\title{
Hybrid phthalocyanine/lead sulphide nanocomposite for bistable memory switches
}

\author{
Zahra Khozaee ${ }^{1}$, Lydia Sosa-Vargas ${ }^{2}$, Andrew N. Cammidge ${ }^{2}$, Michael J. Cook ${ }^{2}$ and Asim K. \\ Ray $^{1 *}$ \\ ${ }^{1}$ Centre of Materials Research, Queen Mary, University of London, Mile End Road \\ London, E1 4NS, UK \\ ${ }^{2}$ School of Chemistry, University of East Anglia, Norwich, NR4 7TJ, UK
}

\begin{abstract}
A simple, one-step method is employed to produce, at room temperature, a single layer of an organic-inorganic nanocomposite containing non-aggregated lead sulphide $(\mathrm{PbS})$ quantum dots (QDs) embedded in a 130nm thick solution processed film of the organic semiconductor $6 \mathrm{PcH} 2$ (metal-free, non-peripherally substituted octahexyl phthalocyanine) on indium tin oxide (ITO). The mean size of PbS QDs is found from X-ray diffraction and transmission electron microscopy techniques to be much smaller than the Bohr radius. Further evidence of the quantum confinement effect is provided by a blue shift in the absorption spectrum and the increased band gap of $1.95 \mathrm{eV}$ with respect to bulk $\mathrm{PbS}$. The current-voltage characteristics of the hybrid and pristine $6 \mathrm{PcH} 2$ films, both in a sandwich configuration with the aluminium top electrode, exhibit bistable switching type hysteresis. The on-off ratio of the nanocomposite device is at least three orders of magnitude higher than that for $6 \mathrm{PcH} 2$ organic films, while both devices operate at a very low bias voltage of $0.5 \mathrm{~V}$. The inclusion of the $\mathrm{PbS} \mathrm{QDs}$ into the $6 \mathrm{PcH} 2$ film enhances the conductivity by nearly two orders of magnitude over that of comparable pristine $6 \mathrm{PcH} 2$ film due to the formation of a charge transfer complex with $\mathrm{PbS}$ QDs and 6PcH2 acting as acceptors and donors of electrons, respectively.
\end{abstract}

Keywords: Nanocomposite, discotic, macrocyclic, octahexylphthalocyanine, non-volatile memory, quantum confinement.

* Present address: Institute of Materials and Manufacturing, Brunel University, Uxbridge, Middlesex UB8 3PH, UK 


\section{Introduction}

Hybrid nanocomposite materials comprising inorganic quantum dots (QD) in an organic matrix are an emerging class of new materials attracting in recent years immense fundamental and applied research interest and effort [1,2]. These materials exhibit significant promise for a variety of applications including sensors [3, 4], displays [5, 6], photovoltaics [7, 8], photonic devices $[9,10]$ and non-volatile memory devices $[11,12]$. The memory devices consist of single or triple active nanocomposite layers sandwiched between two terminals; single layer architecture is, however, considered to be suitable for high density data storage. Both types of memory devices utilise both metallic (gold, silver) and semiconducting QDs (zinc oxide, cadmium sulphide, cadmium selenide and so on) within different phases including colloids, sol-gels and solid films [13, 14]. Low cost solution processing and ink-jet methods can be employed to manufacture these devices over large area flexible substrates, offering advantages over conventional dynamic random access memories, hard disc drives in terms of refresh requirement and degree of responses, respectively [15]. The performance of the single layer memory switches depends upon the size and type of QDs, organic matrix and process parameters. Surfactants are generally used as capping agents to control the aggregation of dots [16], displaying lognormal distribution of QD sizes depending upon nucleation conditions, diffusion, and reaction between species [17]. The particular behavior of QDs arises from quantum confinement effects when the nanoparticles are smaller than the exciton Bohr radius [18]. The device based upon a $180 \mathrm{~nm}$ thick spin-coated hybrid layer of $6 \% \mathrm{wt}$ of colloidal zinc oxide nanoparticles (18nm in size) in polyvinylpyrrolidone (PVP) sandwiched between the indium tin oxide substrate and aluminium top electrode displays non-volatile memory characteristics, giving on/off ratio of $3 \times 10^{3}$ [19]. Switches made of colloidal $5 \mathrm{~nm}$ diameter $\mathrm{ZnO}$ QDs in polymethylmethacryalate (PMMA) within the similar electrode configuration, however, exhibit higher ratio by one order of magnitude [20] The filamentary conduction in a biopolymer silk protein film incorporating gold nanoparticles is found to be responsible for a high value of on/off ratio in the order of $10^{6}$ [21]. Apart from the filamentary conduction model, a trap-filled limited conduction model has been developed using inorganic semiconducting nanoparticles as trapping centres whereas nanoparticles are regarded as being acceptor centres of electrons in the charge complex structure of a hybrid nanocomposite [22]. In order to achieve higher storage density and longer retention time than the silicon flash memory, the bottom gate pentacene transistor was fabricated with the hybrid nanocomposite bilayer of graphene oxide and gold nanoparticles (10nm in average size) as a floating gate. 
This transistor shows non-volatile memory characteristics of high on/off ratio in the order of $10^{4}$ due to high trapping density in the gate for both electrons and holes [23].

Phthalocyanines $(\mathrm{Pc})$ are chemically stable, non-toxic macrocyclic compounds with an extended aromatic $\pi$-system responsible for physically interesting properties leading to a vast range of applications in emerging information technologies [24]. By way of examples, gold and aluminium nanoparticles have been blended into solvent deposited thin films of copper phthalocynine, forming well-organised hybrid nanocomposites for memory devices [25, 26]. Transistor flash memories using perfluorinated copper phthalocyanine and gold nanoparticles in self-assembled polystyrene-block-poly-4-vinylpyridine block copolymer as active and floating layers, respectively, are reported to have shown large charge capacity, stable and programmable-erasable properties with the value in the range of $10^{3}$ and $10^{4}$ for the on-off ratio [27]. However, reported investigations of hybrid memory devices incorporating semiconductor quantum dots in a phthalocyanine matrix are relatively few, in part because of inherent difficulties with thermal evaporation. Phthalocyanine compounds with lipophilic substituents are soluble in common organic solvents and exhibit discotic liquid crystalline behaviour with the cores stacking along columns provide a route to employ these molecules in a cost-effective fabrication of organic electronic devices such as high performance organic thin film transistors as reported [28].

This article reports the bistable switching characteristics of a hybrid nanocomposite containing $\mathrm{PbS}$ quantum dots embedded in a single layered spin coated thin film matrix of a solution processable substituted metal-free discotic liquid crystalline phthalocyanine [29] - nonperipherally substituted octahexylphthalocyanine - Figure 1(a), referred to hereafter as $6 \mathrm{PcH} 2 / \mathrm{PbS}$ in a sandwich configuration between an indium tin oxide (ITO) substrate and aluminium ( $\mathrm{Al}$ ) metal top electrode. This $6 \mathrm{PcH} 2 / \mathrm{PbS}$ hybrid nanocomposite was produced by exploiting the known lability of the lead ion in certain lead phthalocyanine derivatives under mildly acidic conditions [30]. Lead sulphide $(\mathrm{PbS})$ has a large exciton Bohr radius $(18 \mathrm{~nm})$ and shows a strong confinement effect in zero-dimension and this, together with the relative ease of obtaining $\mathrm{PbS}$ nanoparticles smaller than $18 \mathrm{~nm}$, renders it an attractive candidate for forming a direct ligand coupling with $\pi$-conjugated $6 \mathrm{PcH} 2$ molecules for enhanced charge transport [31]. Transmission electron microscopic and X-Ray diffraction studies were performed to measure the orientation and size of $\mathrm{PbS}$ QDs while the bandgap of the dots was estimated from visible optical absorption spectra. The article concludes with an interpretation 
of bistable switching in terms of the transfer of charges between PbS QDs and surrounding $6 \mathrm{PcH} 2$ matrix.

\section{Experimental}

$6 \mathrm{PbPc}$ and pristine $6 \mathrm{PcH} 2$ compounds, the synthesis of which was reported earlier, were used in this investigation [32]. Spin coated films of both compounds were deposited onto ultrasonically cleaned substrates from spreading solutions in toluene, concentration of 10 $\mathrm{mg} / \mathrm{ml}$, using a KW-4A spin-coater (Chemat Technology Inc.) operating at $1000 \mathrm{rpm}$ for 30s. Under these conditions, deposited films were found to be approximately 130nm thick. Following the serendipitous observation described before [33], the nanocomposite $6 \mathrm{PcH} 2 / \mathrm{PbS} /$ films were produced through a twenty four hour exposure of spin-coated films of $6 \mathrm{PbPc}$ to a hydrogen sulphide $\left(\mathrm{H}_{2} \mathrm{~S}\right)$ environment in a sealed container. Under these conditions the lead ion is removed and $\mathrm{PbS}$ nanoparticles form within a film of $6 \mathrm{PcH} 2$ as depicted in Figure 1(a).

Transmission electron microscopic images were obtained using a Jeol Jem-2010 microscope to determine the size of the PbS nanoparticles. The TEM sample was prepared in a powder-like form by carefully removing a sample of the material from the nanocomposite film deposited on ITO. The fragments were then dispersed in distilled water and placed on top of a $3 \mathrm{~mm}$ diameter copper-coated grid. An aluminium thin foil was used to perform the instrument calibration using $80 \mathrm{~cm}$ camera length at $200 \mathrm{KV}$ accelerating voltage (fitted with $\mathrm{LaB}_{6}$ filament). Drop cast films of $6 \mathrm{PcH} 2 / \mathrm{PbS}$ and $6 \mathrm{PcH} 2$, thicker than spin coated films and therefore more appropriate for X-Ray diffraction (XRD) studies, were prepared on silicon wafer substrates. XRD patterns were obtained using a Siemens D5000 X-Ray Diffractometer equipped with $\mathrm{CuK} \alpha$ radiation of wavelength $\lambda_{\mathrm{CuK} \alpha}=0.1540 \mathrm{~nm}$. A Perkin Elmer lambda 950 Spectrometer was employed to study the optical absorption of the nanocomposite on top of the quartz substrates. The selected wavelength ranged between $350 \mathrm{~nm}$ and $900 \mathrm{~nm}$ and recorded at $10 \mathrm{~nm}$ per second.

Using a Keithley 4200-SCS semiconductor characterisation system, the current-voltage characteristics were studied for the single-layered nanocomposite film sandwiched between an indium tin oxide (ITO) substrate and a $60 \mathrm{~nm}$ thick evaporated aluminium (Al) counter electrode as shown in Figure 1(b). The effective area of the devices was defined to be $4.5 \mathrm{~mm}^{2}$ from the overlap between top Al and the bottom ITO electrodes. A similar sandwich structure 
device was also fabricated using $6 \mathrm{PcH} 2$ as the semiconducting active material. The instrument was provided with a preamplifier which reduces the effects of noise in very low current measurement (in the range of $\mathrm{pA}$ ). The voltage was swept in the range of $\pm 2 \mathrm{~V}$ with the scan rate of $0.05 \mathrm{~V} / \mathrm{s}$. In all measurements, the bias was applied to ITO with respect to Al.

\section{Results and Discussion}

\subsection{Transmission electron microscopy}

The formation of nanoparticles with an average size of $4.5 \mathrm{~nm}$ is clearly observed in the TEM images in Figure 2(a) of the $6 \mathrm{PcH} 2 / \mathrm{PbS}$ nanocomposite. The size is far smaller than the exciton Bohr radius of $\mathrm{PbS}$, i.e. $18 \mathrm{~nm}$, confirming they are quantum dots. The rigid environment of the $6 \mathrm{PcH} 2$ solid film is believed to be responsible for limiting the aggregation of the $\mathrm{PbS} \mathrm{QDs}$ and preventing the formation of larger particles. The $\mathrm{PbS} \mathrm{QDs}$ are approximately spherical in shape and dispersed separately from each other. The QDs produced are single crystallites but each are individually orientated in a different direction, as evidenced from the high resolution image in Figure 2(b). The diffraction pattern from the selected-area of the nanocomposite in Figure 2(c) shows the two concentric circles with bright spots which are indicative of two sets of crystal planes. Values of $0.29 \mathrm{~nm}$ and $0.21 \mathrm{~nm}$ were estimated for the lattice spacing d using the relation:

$$
\mathrm{rd}=\mathrm{C}
$$

where $\mathrm{r}$ is the radius of the selective-area diffraction pattern. $\mathrm{C}=1.9815 \times 10^{6}$ and is defined as the camera constant for a TEM operating voltage of $200 \mathrm{kV}$. Lattice spacing values correspond to the (200) and (220) PbS crystal planes.

\subsection{X-Ray diffraction}

The X-Ray diffraction spectrum in Figure 3 of the nanocomposite $6 \mathrm{PcH} 2 / \mathrm{PbS}$ film supported on a silicon wafer shows three peaks at $25.96^{\circ}, 30.03^{\circ}$ and $43.08^{\circ}$. These peaks are identified as the 111, 200 and 220 crystal planes of $\mathrm{PbS}$, according to the standard database of PbS crystals [34]. The occurrence of more than one Bragg peak of $\mathrm{PbS}$ implies that the formed $\mathrm{PbS}$ nanocrystals are orientated in different directions. This observation is consistent with the information obtained from the TEM images. The average particle size was calculated to be 5.9 $\pm 0.5 \mathrm{~nm}$ from the half-width $\beta$ of diffraction peak, using Scherrer equation:[35] 


$$
\mathrm{D}=\frac{\mathrm{k} \lambda_{\mathrm{CuK} \alpha}}{\beta \cos \theta}
$$

where $\mathrm{k}$ is the geometric factor (usually assumed to be 0.9 ). The size of the nanoparticles is in good agreement with that observed in the TEM images. The XRD spectra of pure $6 \mathrm{PcH} 2$ and $6 \mathrm{PbPc}$ films are also included for the sake of comparison. The Bragg peaks observed for the nanocomposite at small angles $2 \theta<25^{\circ}$ are believed to be associated with those of $6 \mathrm{PcH} 2$ and residual $6 \mathrm{PbPc}$ assemblies.

\subsection{UV-Vis spectroscopy}

Visible absorption spectra of the spin coated films of $6 \mathrm{PbPc}$, the $6 \mathrm{PcH} 2 / \mathrm{PbS}$ nanocomposite and pristine $6 \mathrm{PcH} 2$ on quartz substrates are depicted in Figures 4(a), (b) and (c) respectively. As expected, there is a resemblance between the spectra of the nanocomposite and pristine $6 \mathrm{PcH} 2$ confirming the formation of $6 \mathrm{PcH} 2$ in the nanocomposite. The spectrum in Figure 4(d) which was obtained from the difference between the two curves in Figure 4(a) and (b) shows a peak at around $600 \mathrm{~nm}$ which is identified with the first excitonic peak of $\mathrm{PbS}$ QDs from the observation of the trend of increasing intensity with decreasing wavelength less than $600 \mathrm{~nm}$. The blue shift in absorption is large compared to that in the spectrum of bulk material absorption onset at about $3200 \mathrm{~nm}$ indicating the band gap modification of the QDs due to the quantum confinement effect [36]. The three broad peaks at $480 \mathrm{~nm}, 700 \mathrm{~nm}$ and $780 \mathrm{~nm}$ are believed to be related to the presence of residual $6 \mathrm{PbPc}$ molecules within the nanocomposite film.

The dependence of the absorbance $A$ in Figure 4(d) on the incident photon energy $h v$ is usually described in terms of the Tauc law $h v$ in the form:[37]

$$
A \propto \frac{1}{h v} \sqrt{\left(h v-E_{g}\right)}
$$

The plot of $(A h v)^{2}$ versus incident photon energy $h v$ in the inset is linear, implying that electronic transitions in $\mathrm{PbS}$ QDs are direct. The band gap $\mathrm{E}_{\mathrm{g}}$ of the PbS QDS is found to be $1.95 \mathrm{eV}$ from the intercept on the abscissa of the plot. This value is much larger than the bulk band gap of $0.41 \mathrm{eV}$, indicating the occurrence of the quantum confinement in monodispersed QDs in the $6 \mathrm{PcH} 2 / \mathrm{PbS}$ nanocomposite. A value of $2.55 \mathrm{eV}$ for $\mathrm{E}_{\mathrm{g}}$ is estimated for $\mathrm{PbS}$ QDs having a distribution of sizes in the range of $5 \mathrm{~nm}-10 \mathrm{~nm}$ incorporated in a spin-coated PVP matrix [38]. Using the effective mass approximation model [18], the average $\mathrm{PbS}$ QDS 
diameter is estimated to be about $3.7 \mathrm{~nm}$. This value is in reasonable agreement with those obtained by TEM $(\sim 4.5 \mathrm{~nm})$ and XRD $(\sim 5.9 \mathrm{~nm})$ techniques.

\subsection{Bistable electrical behaviour}

Figure 5 shows anticlockwise electrical hysteresis behaviour in the current-voltage $\mathrm{I}(\mathrm{V})$ characteristics of both the nanocomposite $6 \mathrm{PcH} 2 / \mathrm{PbS}$ and $6 \mathrm{PcH} 2$ films sandwiched between the ITO and Al electrodes during the voltage cycling between $\pm 2 \mathrm{~V}$. This observation corresponds to the essential features of a two terminal bistable switch. The first sweep from $0 \mathrm{~V}$ to $+2 \mathrm{~V}$ is included in the inset and the current through the $6 \mathrm{PcH} 2 / \mathrm{PbS}$ film is found to be higher than that through the $6 \mathrm{PcH} 2$ film by about two orders of magnitude, giving corresponding values of $3.1 \times 10^{-10} \Omega^{-1} \mathrm{~m}^{-1}$ and $6.0 \times 10^{-12} \Omega^{-1} \mathrm{~m}^{-1}$ for Ohmic conductivity in the voltage range $<0.5 \mathrm{~V}$.

The switch-on voltage $\mathrm{V}_{\text {on }}=1.7 \mathrm{~V}$ to the $\mathrm{ON}$ (high-current) state for the $6 \mathrm{PcH} 2 / \mathrm{PbS}$ film was found to be lower than $\mathrm{V}_{\text {on }}=2.1 \mathrm{~V}$ for the $6 \mathrm{PcH} 2$ film, indicating significantly lower power consumption by the nanocomposite. The $6 \mathrm{PcH} 2 / \mathrm{PbS}$ film was also switched to the OFF (lowcurrent) state at $\mathrm{V}_{\text {off }}=-0.9 \mathrm{~V}$ which is lower than $\mathrm{V}_{\text {off }}=-1.6 \mathrm{~V}$ for the $6 \mathrm{PcH} 2$ film. The transition from the OFF state to the $\mathrm{ON}$ state is equivalent to the process of writing a digital memory cell and both devices remained stable in the ON state even after the power is turned off, displaying the important characteristic of a non-volatile memory. The OFF state is identified with erasing the information from the digital memory cell.

The percolation limit can be achieved only if the nanoparticles concentration is high, usually in volume fraction higher than $15-20 \%$ [39]. The volume fraction $(x)$ of the PbS QDs in this nanocomposite is estimated to be $3 \%$ from the formula in the form [40]

$$
x=\frac{\frac{w}{\rho^{Q D}}}{\frac{w}{\rho^{Q D}}+\frac{(1-w)}{\rho^{P c}}}
$$

where $w=M^{Q D} /\left(M^{Q D}+M^{P c}\right)$ is the mass fraction of the PbS QDs, $M^{Q D}=239.27 \mathrm{~g} \cdot \mathrm{mol}^{-1}$ and $M^{P c}=1187.83$ g.mol ${ }^{-1}[\mathbf{c}]$ are the molecular weights of $\mathrm{PbS}$ and $\mathrm{C}_{6} \mathrm{H}_{2} \mathrm{Pc}$, respectively, and $\rho^{Q D}=7.60 \mathrm{~g} \cdot \mathrm{cm}^{-3}[34]$ and $\rho^{P c}=1.121 \mathrm{~g} \cdot \mathrm{cm}^{-3}$ are the densities of $\mathrm{PbS}$ and $\mathrm{C}_{6} \mathrm{H}_{2} \mathrm{Pc}$, respectively. The $\mathrm{PbS}$ QDs are dispersed among the $6 \mathrm{PcH} 2$ matrix and therefore the formation 
of continuous conducting paths between the electrodes is not regarded as being responsible for charge transport in this single layered nanocomposite.

The bistable switching effect in a nanocomposite device may be interpreted on the basis of optical absorption data in Section 3.3. Since the effective masses of holes and electrons are both approximately equal to $0.085 \mathrm{~m}_{0}$ where $\mathrm{m}_{0}$ is the rest mass of electrons in $\mathrm{PbS}$, there will be equal shifts of the QD frontier energy levels from the bulk valence and conduction band edges at $4.55 \mathrm{eV}$ and $4.96 \mathrm{eV}$, respectively due to the quantum confinement [41]. The highest occupied molecular orbital (HOMO) and lowest unoccupied molecular orbital (LUMO) levels of the $\mathrm{PbS}$ QDs are, therefore, believed to have located at $5.73 \mathrm{eV}$ and $3.78 \mathrm{eV}$. These observations are found to be consistent with the $\mathrm{PbS}$ particle size determined from the $\mathrm{X}$-Ray and TEM studies.

As shown in Figure 6, the HOMO energy of the PbS QDs is lower than that of the neighbouring $6 \mathrm{PcH} 2$ molecules [42]. Under these circumstances, the $\mathrm{PbS}$ QDs act as preferential acceptor centres of electrons while the $6 \mathrm{PcH} 2$ donor molecules provide the supply of electrons. Electrons are progressively transferred to $\mathrm{PbS}$ QDs from adjacent $6 \mathrm{PcH} 2$ molecules under the influence of the applied voltage and remain trapped therein. This charge transfer leads to a significant increase in the density of the majority carriers (holes) in the $6 \mathrm{PcH} 2$ matrix. As a result, the nanocomposite film is formed into a complete charge transfer complex after the application of a positive bias voltage larger than $\mathrm{V}_{\text {on }}$ with simultaneous reduction in recombination probability for both types of carriers. The electrons in the PbS QDs are prevented from returning to the surrounding low conductive $6 \mathrm{PcH} 2$ matrix when the voltage is withdrawn, giving rise to the non-volatile feature of the memory device. Electrons remain trapped in PbS QDs under negative bias until $\mathrm{V}_{\text {off }}$ when PbS QDs are likely to empty transferred charges, resulting in transition to the off-state. A similar abrupt transition from high to low conductivity states corresponding to the bias voltages of $+2.8 \mathrm{~V}$ and $-1.8 \mathrm{~V}$, respectively by four orders of magnitude was observed for a polystyrene film consisting of gold nanoparticles and 8-hydroxyquinoline (8HQ) between two aluminium electrodes. This behaviour is believed to be due to an external field induced charge transfer between gold nanoparticles and 8HQ [43].

The on/off ratio i.e., ratio of currents obtained at same voltage for low and high conducting states, which is related to the level of electron occupancy of $\mathrm{PbS}$ quantum dots, is plotted in 
Figure 7 against the bias voltage. The value of this ratio for $6 \mathrm{PcH} 2 / \mathrm{PbS}$ at the voltage of $+0.5 \mathrm{~V}$ is found to be $2.9 \times 10^{4}$. This value is comparable to one obtained at the reading voltage of $1 \mathrm{~V}$ for the spin-coated hybrid films containing colloidal $\mathrm{ZnO}$ QDs of $5 \mathrm{~nm}$ size in polymethylmethacryalate between the ITO substrate and aluminium top electrode [44]. The value of on/off ratio reported for a two terminal device containing graphene quantum dots embedded in the matrix of poly(ethylene glycol) bis(carboxymethyl) ether between two gold electrodes in a planar structure operating at $\pm 4.3 \mathrm{~V}$ is at least three orders of magnitude smaller than the one obtained in the presented investigation [45]. The non-volatile memory effect is also observed in thin film field effect transistors using embedded $\mathrm{PbS}$ nanocrystals between layers of silicon oxide and amorphous fluoropolymer as a floating gate. This transistor device operates at relatively high switch-on and switch-off voltages of $20 \mathrm{~V}$ and $30 \mathrm{~V}$, respectively with the on/off ratio is $10^{3}$ [46]. The sandwich device containing pristine $6 \mathrm{PcH} 2$ also shows memory characteristics in Figure 7 at similar voltages but with a considerably smaller on-off ratio of 31 at the same bias voltage of $0.5 \mathrm{~V}$. As is obvious from the band diagram in Figure 6, the switching property of the $6 \mathrm{PcH} 2$ film without $\mathrm{PbS}$ may be explained in terms of the depletion layer at the ITO/6PcH2 contact. The majority of the traps became filled at voltages above $\mathrm{V}_{\text {on }}$ and injected charges from the electrode were free to participate in the current flow, leading to the sharp increase in the current [47]. Non-volatile resistance switching memory characteristics have also been observed in the conduction characteristics of $20 \mathrm{~nm}$ thick sputtered titanium oxide embedded with nickel nanocrystals of an average size of $4 \mathrm{~nm}$ in sandwich configurations between silicon substrates and platinum top electrode. A value of $5 \times 10^{3}$ for on/off ratio was obtained when the sample film was annealed at $1000^{\circ} \mathrm{C}$ for 5 minutes [48]. A nickel silicide (NiSi) interlayer was sandwiched between two atomic layer deposited hafnium oxide $\left(\mathrm{HfO}_{2}\right)$ to fabricate a memory switch on platinum/titanium/silicon/ silicon dioxide substrate with $20-50 \mathrm{~nm}$ thick e-beam evaporated platinum/titanium as the top electrode. The memory produced on/off ratio in the order of $10^{2}$ when the NiSi interlayer was annealed in $550^{\circ} \mathrm{C}$ for $1 \mathrm{~min}[49]$.

Figure 8 (a) shows the retention of the bistable switching characteristics of $6 \mathrm{PcH} 2 / \mathrm{PbS}$ nanocomposite. As the time delay was increased from $5 \mathrm{~min}$ to15min, a small change in threshold voltage was observed. This may be attributed to the possibility that charges remained trapped in deep traps as the bias was cycled from the positive to negative voltage. The measurements were repeated on the sample that had been stored in the open laboratory 
environment over seven days. Figure 8(b) shows the variation of high resistance $\left(R_{\text {off }}\right)$ and low resistance $\left(R_{o n}\right)$ states recorded for different cycles over that period. Values of $R_{\text {on }}$ and $R_{\text {off }}$ were determined from the slopes of $\mathrm{I}(\mathrm{V})$ characteristics at the bias of $0.5 \mathrm{~V}$. Both $\mathrm{R}_{\text {on }}$ and $\mathrm{R}_{\text {off }}$ states remained well resolved and there was no overlapping between two states, indicating the reasonably stable and non-volatile features of the $\mathrm{ITO} / 6 \mathrm{PcH} 2 / \mathrm{PbS} / \mathrm{Al}$ resistive switch. The devices were also found to be stable in air over a week of monitoring without requiring additional encapsulation. The nanocomposite did not suffer from appreciable degradation due to oxygen adsorption and carrier trapping in the deep states is believed to be small. This type environmentally stable behaviour is observed for mobility measurements in the similarly substituted zinc phthalocyanine derivatives [50].

\section{Conclusions}

An organic-inorganic nanocomposite of lead sulphide $(\mathrm{PbS})$ quantum dots embedded in a nonperipherally octa-hexyl substituted metal-free phthalocyanine film has been successfully prepared at room temperature by a simple and low cost printing-compatible solution process. The size of the $\mathrm{PbS}$ quantum dots was determined from two independent measurements to be less than $6 \mathrm{~nm}$, much smaller than the Bohr radius. The rigid environment of the matrix and the presence of the high molecular weight organic molecules prohibit aggregation of the quantum dots and the use of surfactants is not required. The quantum confinement effect in $\mathrm{PbS}$ shifts the frontier energy levels of the QDs and increases the band gap energy from $0.41 \mathrm{eV}$ in bulk to $1.95 \mathrm{eV}$. This high conductance state of $6 \mathrm{PcH} 2 / \mathrm{PbS}$ nanocomposite film is a very desirable property for developing noise free, fast data readouts in an integrated circuit memory. The nanocomposite also exhibits bistable electrical behaviour with a large on-off ratio of higher than $10^{4}$ giving rise to a very low misreading rate. The low switch-on voltage means low power consumption, contributing to increased reliability. In light of complexities inherent with the device fabrication and structure of these all inorganic memory switches, the performance of the present solution processed, simply configured, environmentally stable and energy efficient memory switch is quite encouraging for developing high density memory system.

\section{Acknowledgements}

The authors are grateful to Professor W. Gillin of Queen Mary, University of London, for fruitful discussions and input.

\section{References}


1. Nicole, L.; Laberty-Robert, C.; Rozes L. and Sanchez, C. 2014 Nanoscale 6(12), 6267-6292.

2. Kumar, S. 2014 NPG Asia Mater. 6, Article Number: e82

3. Wang, S. R.; Kang, Y. F.; Wang, L. W.; Zhang, H. X. Wang, Y.S. and Wang, Y. 2013 Sens. Actuator B-Chem. 182, 467-481.

4. $\quad$ Nguyen T. D. and Tran T. H. 2014 RSC Adv. 4(2), 916-942.

5. Giovanella, U., Leone, G., Galeotti, F., Mroz, W., Meinardi and F. Botta, 2014 Chem. Mat. 26(15), 4572-4578.

6. Liang, R. Z.; Yan, D. P.; Tian, R.; Yu, X. J.; Shi, W. Y.; Li, C. Y.; Wei, M.; Evans, D. G. and Duan, X. 2014 Chem. Mat. 26(8), 2595-2600.

7. Nam, M.; Park, J.; Kim, S. W. and Lee, K. 2014 J. Mater. Chem. A 2(11), 3978-3985.

8. Zhao, L. and Lin, Z.Q. 2012 Adv. Mater. 24(32), 4353-4368.

9. Petti, L.; Rippa, M.; Zhou, J.; Manna, L. and Mormile, P. 2011 Nanotechnology 22(28), Article Number: 285307

10. Jia, B. H.; Buso, D.; van Embden, J.; Li, J. F. and Gu, M. 2010 Adv. Mater. 22(22), 2463-2467.

11. Kim, T. W.; Yang, Y.; Li, F. S. and Kwan, W. L. 2012 NPG Asia Mater. 4, Article Number: e18

12. Das, B. C. and Pal, A. J. 2008 ACS Nano 2(9), 1930-1938.

13. Konstantatos, G. and Sargent, E. H. 2001 Infrared Phys. Technol. 54(3) 278-282.

14. Mitzi, D. B.. 2010 Chem. Mat. 13(10), 3283-3298.

15. Ouyang, J.Y. 2010 Nano Rev. 1, 10.3402

16. Bakshi, M.S.; Thakur, P.; Sachar, S.; Kaur, G.; Banipal, T.S.; Possmayer, F. and Petersen, N.O. 2007 J. Phys. Chem. C 111(49), 18087-18098.

17. de Lamaestre, R. E. and Bernas, H. 2006 Phys. Rev. B, 73(12), Article Number: 125317.

18. Yoffe, A. D. 2001 Adv. Phys. 50(1), 1-208.

19. Onlaor, K..; Thiwawong, T. and Tunhoo, B., 2014 Org. Electron. 15(6), 1254-1262.

20. Son, D. I.; You, C. H.; Kim, W. T.; Jung, J.H. and Kim, T.W. 2009 Appl. Phys. Lett. 94(13), Article Number: 132103.

21. Gogurla, N.; Mondal, S. P.; Sinha, A. K.; Katiyar, A. K.; Banerjee, W.; Kundu, S. C. and Ray, S. K. 2013 Nanotechnology, 24(34), Article Number: 345202. 
22. Ling, Q. D.; Liaw, D. J.; Zhu, C. X.; Chan, D. S. H.; Kang, E. T. and Neoh, K. G. 2008 Prog. Polym. Sci. 33(10), 917-978.

23. Gao, X.; She, X. J.; Liu, C. H.; Sun, Q. J.; Liu, J. and Wang, S. D. 2013 Appl. Phys. Lett. 102(2), Article Number: 023303.

24. Claessens, C. G.; Hahn, U. and Torres, T. 2008 Chem. Rec. 8(2), 75-97.

25. Molodtsova, O.V. Aristova, I. M.; Babenkov, S. V.; Vilkov, O. V and Aristov, V. Y. 2014 J. Appl. Phys. 115(16), Article Number: 164310.

26. Aristov, V. Y.; Molodtsova, O. V.; Laubschat, C.; Zhilin, V. M.; Aristova, I. M.; Kveder, V. V. and Knupfer, M. 2010 Appl. Phys. Lett. 97(11), Article Number: 113103.

27. Leong, W. L.; Mathews, N.; Mhaisalkar, S.; Lam, Y. M.; Chen, T. P. and Lee, P. S. 2009 J. Mater. Chem. 19(39), 7354-7361.

28. Chaure, N. B.; Pal, C.; Barard, S.; Kreouzis, T.; Ray, A. K.; Cammidge, A. N.; Chambrier, I.; Cook, M. J.; Murphy, C. E. and Cain, M. G. 2012 J. Mater. Chem. 22(36), 19179-19189.

29. Cook, M. J.; Daniel, M. F.; Harrison, K. J.; Mckeown, N. B. and Thomson, A. J. 1987 J. Chem. Soc.-Chem. Commun. 14, 1086-1088.

30. Sosa-Vargas, L.X.; Chambrier, I.; MacDonald, C. J.; Coles, S. J.; Tizzard, G. J.; Cammidge, A. N. and Cook, M.J. 2013 J. Porphyr. Phthalocyanines 17( 6-7). 511521

31. Park Y. and Advincula R.C. 2011 Chem. Mat. 23(19), 4273-4294.

32. Burnham, P. M.; Chambrier, I. ; Hughes, D. L.; Isare, B.; Poynter, R. J.; Powell, A. K. and Cook, M. J. 2006 J. Porphyr. Phthalocyanines 10(9-10), 1202-1211.

33. Nabok, A. V.; Ray, A. K.; Cook, M. J.; Burnham, P. M.; Iwantono, Yanuar, H.; Simmonds, M. and Basova, T. V. 2004 IEEE Trans. Nanotechnol. 3(3), 388-394.

34. McMurdie, H. F.; Morris, M.C.; Evans, E. H.; Paretzkin, B.; Wong-Ng, W. and Hubbard, C.R. Standard X-Ray Diffraction Powder Patterns from The JCPDS Research Associateship. Powder Diffraction. 1986, 1, 265-275.

35. Patterson, A. 1939 Phys. Rev. 56(10), 978-982.

36. Wang, Y.; Suna, A.; Mahler, W and Kasowski, R. 1987 J. Chem. Phys. 87(12), 73157322.

37. Ray, A.K. and Hogarth, C. A. 1990 J. Phys. D-Appl. Phys. 23(4), 458-459.

38. Patel, J. D. and Chaudhuri, T. K. 2009 Mater. Res. Bull. 44(8), 1647-1651. 
39. Bakueva, L.; Musikhin, S.; Sargent, E. H., Ruda H. E. and Shik, A. "Luminescence and photovoltaic effects in polymer-based nanocomposites," Handbook of organicinorganic hybrid materials and nanocomposites, H. S. Nalwa (Editor), vol. 2, American Scientific, 2003, p. 181.

40. Choudhury, K. R.; Samoc, M.; Patra A. and Prasad, P. N. 2004 J. Phys. Chem. B 108(5), 1556-1562.

41. Kang, I. and Wise, F. W. 1997 J. Opt. Soc. Am. B-Opt. Phys. 14 (7), 1632-1646.

42. Alfredsson, Y.; Brena, B.; Nilson, K.; Ahlund, J. Kjeldgaard, L.; Nyberg, M.; Luo, Y.; Martensson, N.; Sandell, A.; Puglia, C. and Siegbahn, H.. J. 2005 Chem. Phys. 122(21) Article Number: 214723.

43. Ouyang, J.Y.; Chu, C.W.; Szmanda, C.R.; Ma, L.P. and Yang, Y. 2004 Nat. Mater. 3(12), 918-922.

44. Son, D. I.; You, C. H.; Jung, J. H. and Kim, T. W. 2010 Appl. Phys. Lett. 97(1), Article Number: 013304.

45. Mihalache, I.; Radoi, A.; Munteanu, C.; Kusko M. and Kusko, C. 2014 Org. Electron. 15(1), 216-225

46. Chung, D.S.; Kang, I.; Kwon, S. K. and Kim, Y. H. $2013 \operatorname{RSC} A d v .3(32), 13156-$ 13162 .

47. Mukherjee, B.; Ray, A. K.; Sharma, A. K., Cook, M. J. and Chambrier, I, 2008 J. Appl. Phys, 103(7), Article Number: 074507

48. Panda, D.: Huang, C.-Y. and Tseng T. Y. 2012 Appl. Phys. Lett. 100, 112901.

49. Panda, D.: Dhar, A. and Ray, S. K. 2012 IEEE Trans. Nanotechnol., 11(1), 51-55.

50. Chaure, N. B.; Barard, S.; Ray, A. K.; Cammidge, A. N and Cook, M. J.; 2013 EPL 104(5), Article Number: 57005 


\section{Figure captions}

Figure 1 (a) 1,4,8,11,15,18,22,25-Octakis(hexyl) substituted phthalocyanines showing the chemical structures of the lead metallated derivative, $(6 \mathrm{PcPb})$, and the metal-free analogue $6 \mathrm{PcH} 2$ within a scheme illustrating the formation of lead sulphide $(\mathrm{PbS})$ nanoparticles, (b) Sandwich device structure of $6 \mathrm{PcH} 2 / \mathrm{PbS}$ nanocomposite between ITO and Al electrodes.

Figure 2 TEM images of the $6 \mathrm{PcH} 2 / \mathrm{PbS}$ nanocomposite with magnification of (a) $15 \mu \mathrm{m}$ and (b) $5 \mu \mathrm{m}$. The darker phases are the $\mathrm{PbS}$ quantum dots. (c) The selected-area diffraction pattern from the $6 \mathrm{PcH} 2 / \mathrm{PbS}$ nanocomposite. The two bright concentric circuits are related to the 200 and 220 crystal planes of $\mathrm{PbS}$.

Figure $3 \mathrm{X}$-Ray diffraction patterns from the $6 \mathrm{PcH} 2 / \mathrm{PbS}$ nanocomposite. The observed Bragg peaks corresponding to $\mathrm{PbS}$ crystal planes have been marked by solid lines. Those for films of pristine $6 \mathrm{PcH} 2$ are shown by dotted lines and those for $6 \mathrm{PcPb}$ alone by dashed lines.

Figure 4 Optical absorbance spectra of: (a) 6PcPb film; (b) 6PcH2/PbS nanocomposite; (c) pristine $6 \mathrm{PcH} 2$ scaled down by a factor of 0.85 ; and (d) the result of subtraction of curve (b) from curve (c).

Figure 5 The bistable electrical behaviour of $6 \mathrm{PcH} 2 / \mathrm{PbS}$ nanocomposite (Closed traingles) and pristine $6 \mathrm{PcH} 2$ (Open rectangles) sandwiched between ITO and $\mathrm{Al}$ electrodes. The arrows show the direction of sweeping voltage

Figure 6 Energy level diagram of $6 \mathrm{PcH} 2 / \mathrm{PbS}$ nanocomposite sandwiched between ITO and $\mathrm{Al}$ electrodes. The values are based on the frontier energy levels of the PbS QDs, the reported energy levels for metal-free phthalocyanine in the literature. ${ }^{35}$

Figure 7 The on/off ratio of two-terminal switches containing 6 $\mathrm{PcH} 2 / \mathrm{PbS}$ nanocomposite. (triangles) and pristine $6 \mathrm{PcH} 2$ film (circles).

Figure 8 (a) Bistable behaviour of $6 \mathrm{PcH} 2 / \mathrm{PbS}$ nanocomposite for the first cycle (triangle), after 5minutes (circle), 15 minutes (square) and 3 days (cross); (b) Endurance 
characteristic of $6 \mathrm{PcH} 2 / \mathrm{PbS}$ nanacomposite (solid rectangles and circles for On and Off states respectively. 


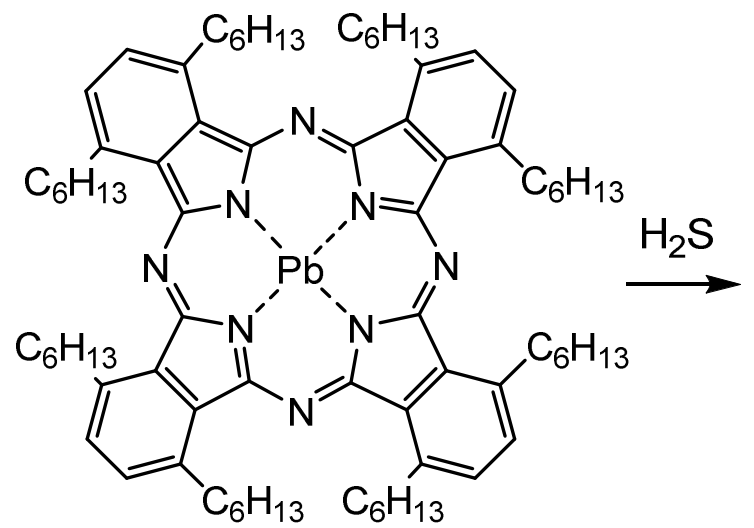

(a)

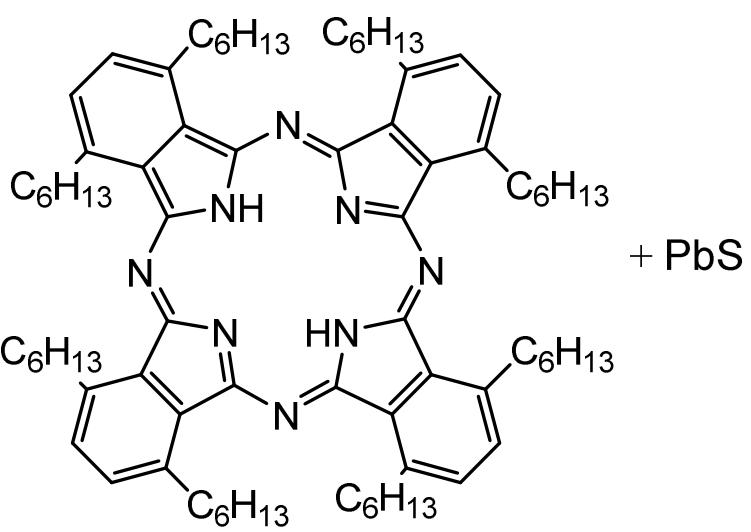

$6 \mathrm{PcH} 2$

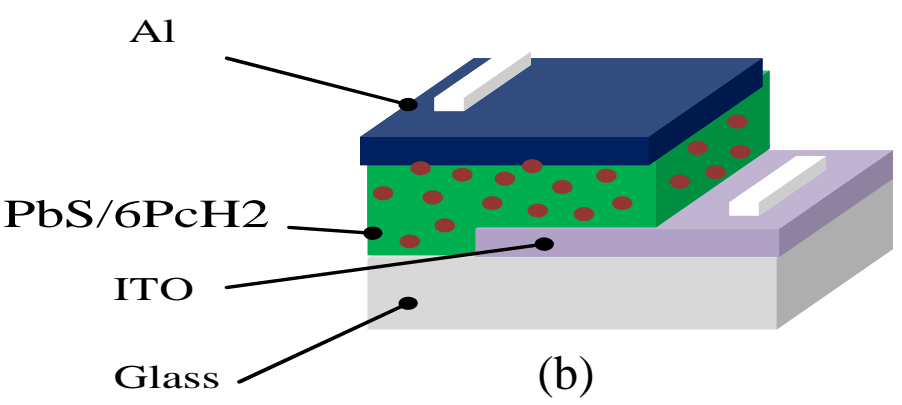

Figure 1 (a) 1,4,8,11,15,18,22,25-Octakis(hexyl) substituted phthalocyanines showing the chemical structures of the lead metallated derivative, $(6 \mathrm{PcPb})$, and the metal-free analogue $6 \mathrm{PcH} 2$ within a scheme illustrating the formation of lead sulphide $(\mathrm{PbS})$ nanoparticles, (b) Sandwich device structure of $6 \mathrm{PcH} 2 / \mathrm{PbS}$ nanocomposite between ITO and Al electrodes 


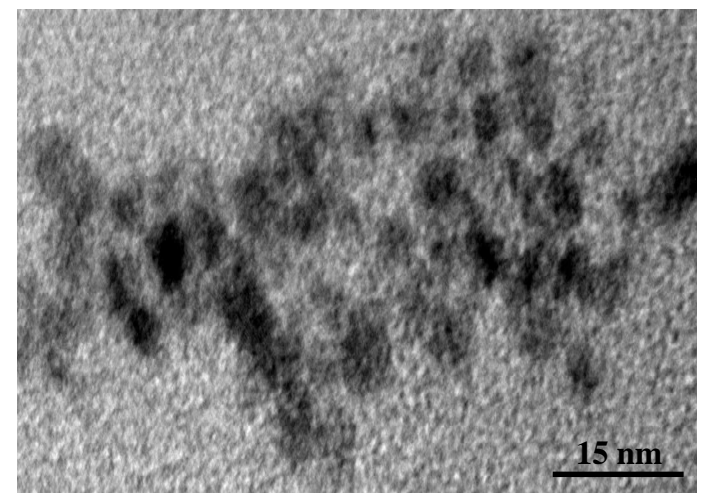

(a)

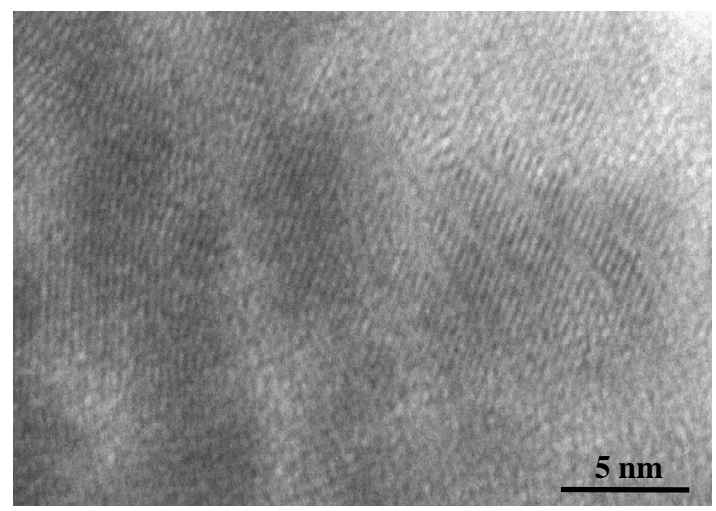

(b)

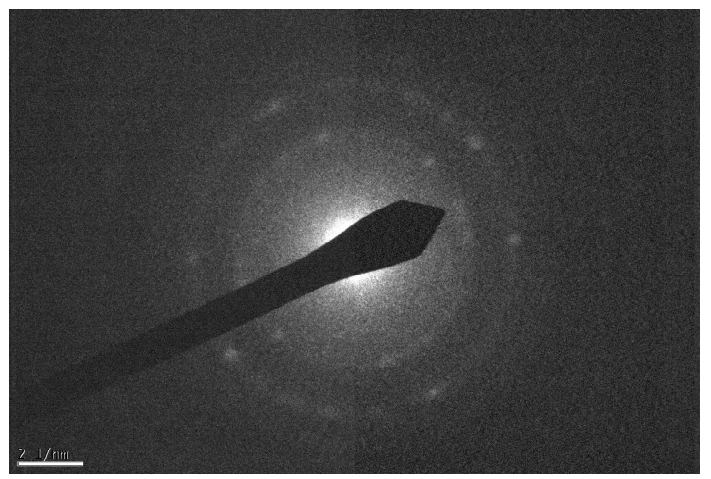

(c)

Figure 2 TEM images of the $6 \mathrm{PcH} 2 / \mathrm{PbS}$ nanocomposite with magnification of (a) $15 \mu \mathrm{m}$ and (b) $5 \mu \mathrm{m}$. The darker phases are the PbS quantum dots. (c) The selected-area diffraction pattern from the $6 \mathrm{PcH} 2 / \mathrm{PbS}$ nanocomposite. The two bright concentric circuits are related to the 200 and 220 crystal planes of $\mathrm{PbS}$. 


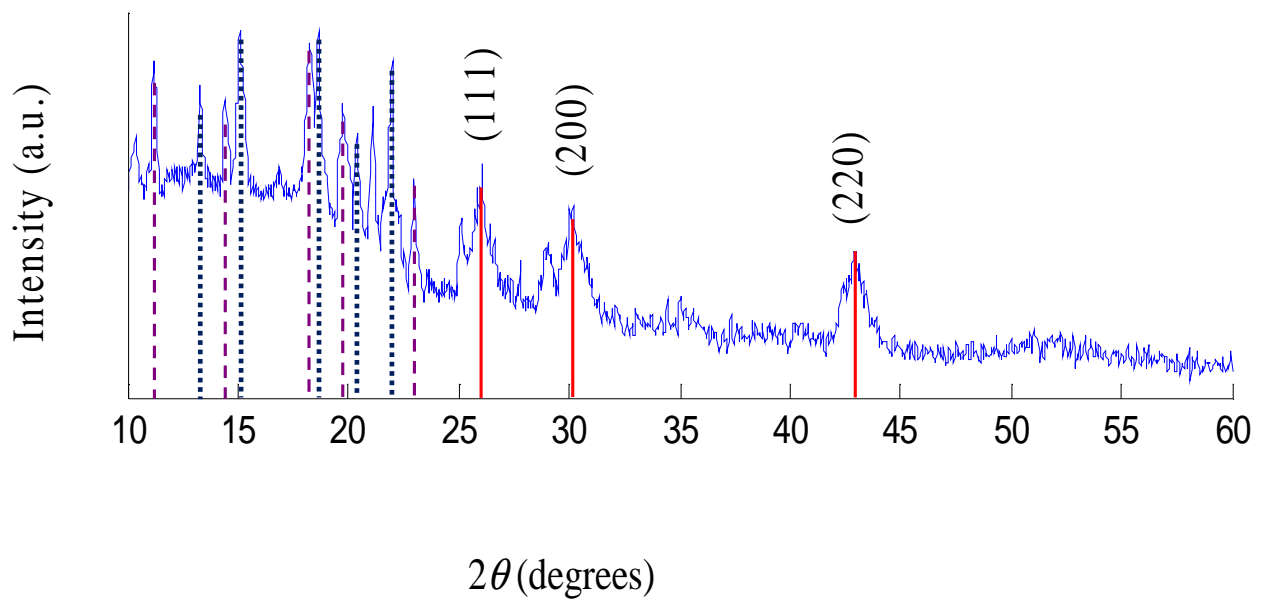

Figure $3 \mathrm{x}$-Ray diffraction patterns from the $6 \mathrm{PcH} 2 / \mathrm{PbS}$ nanocomposite. The observed Bragg peaks corresponding to $\mathrm{PbS}$ crystal planes have been marked by solid lines. Those for films of pristine $6 \mathrm{PcH} 2$ are shown by dotted lines and those for $6 \mathrm{PcPb}$ alone by dashed lines. 


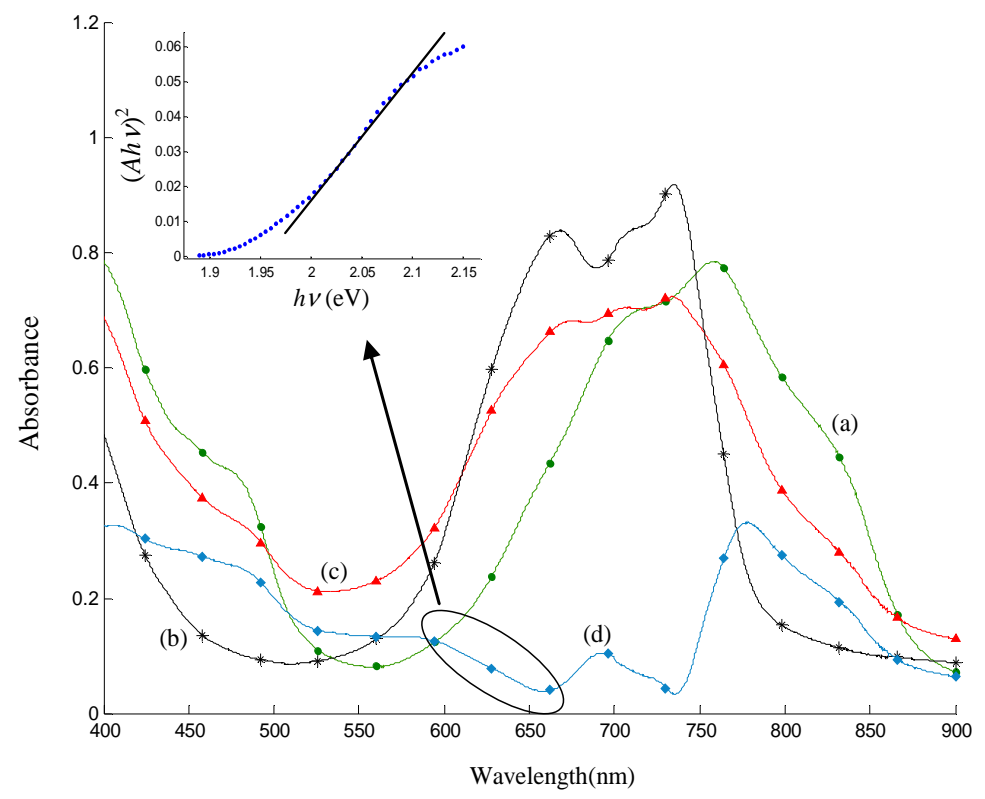

Figure 4 Optical absorbance spectra of: (a) 6PcPb film; (b) 6PcH2/PbS nanocomposite; (c) pristine $6 \mathrm{PcH} 2$ scaled down by a factor of 0.85 ; and (d) the result of subtraction of curve (b) from curve (c). 


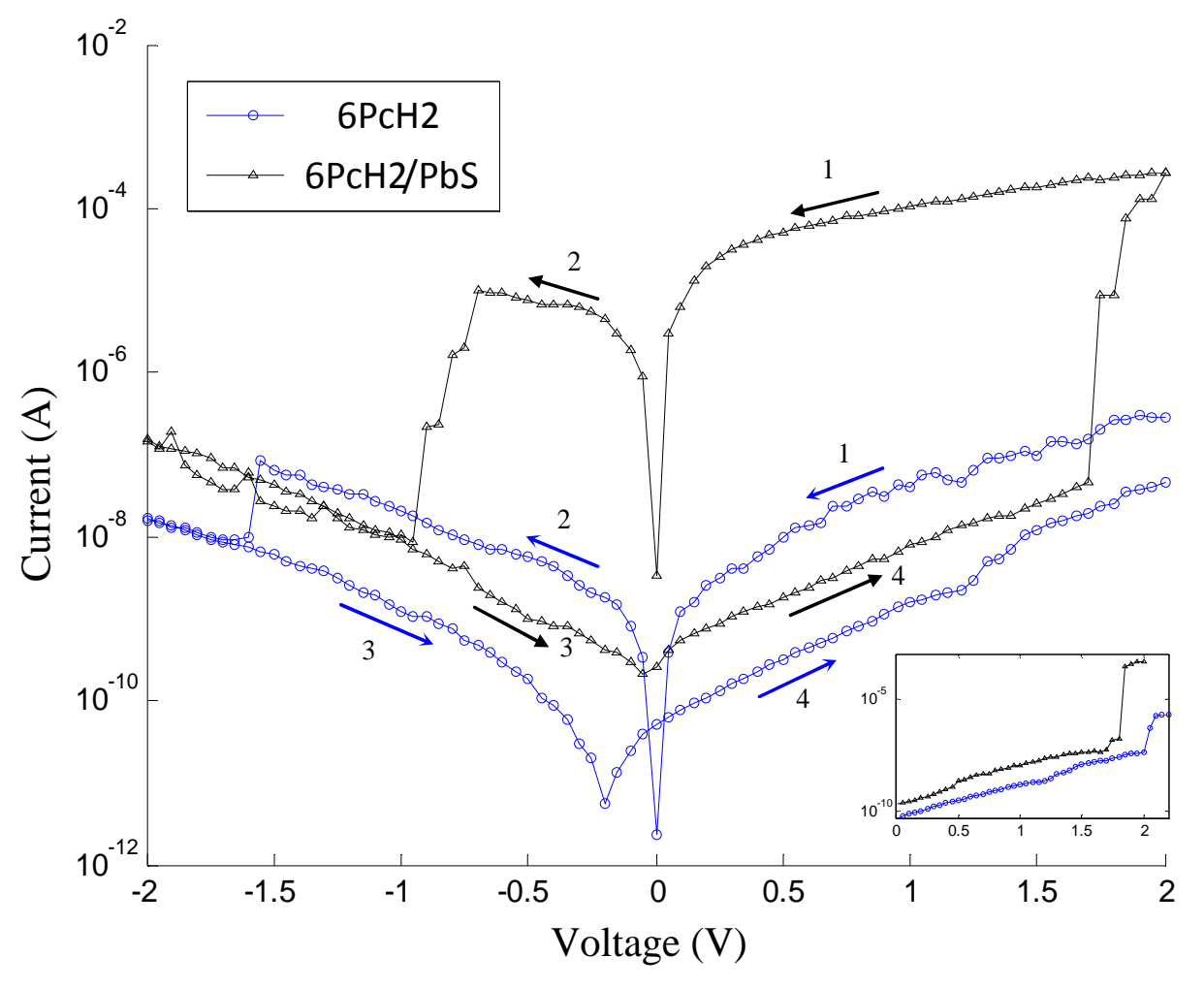

Figure 5 The bistable electrical behaviour of $6 \mathrm{PcH} 2 / \mathrm{PbS}$ nanocomposite (Traingles) and pristine $6 \mathrm{PcH} 2$ (Circles) sandwiched between ITO and $\mathrm{Al}$ electrodes. The arrows show the direction of sweeping voltage 


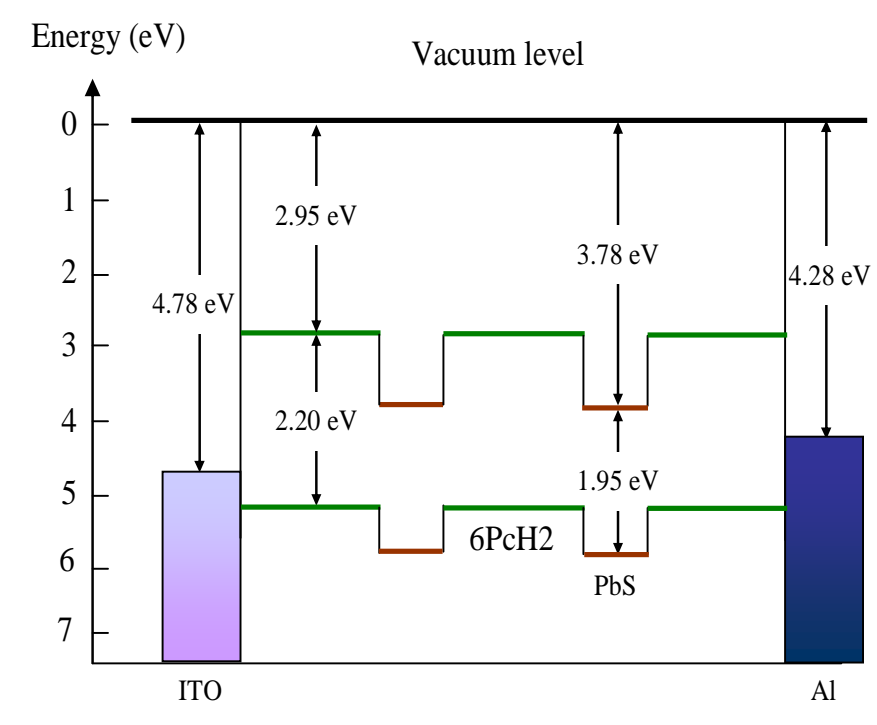

Figure 6 Energy level diagram of $6 \mathrm{PcH} 2 / \mathrm{PbS}$ nanocomposite sandwiched between ITO and $\mathrm{Al}$ electrodes. The values are based on the frontier energy levels of the PbS QDs, and the reported energy levels for metal-free phthalocyanine [42]. 


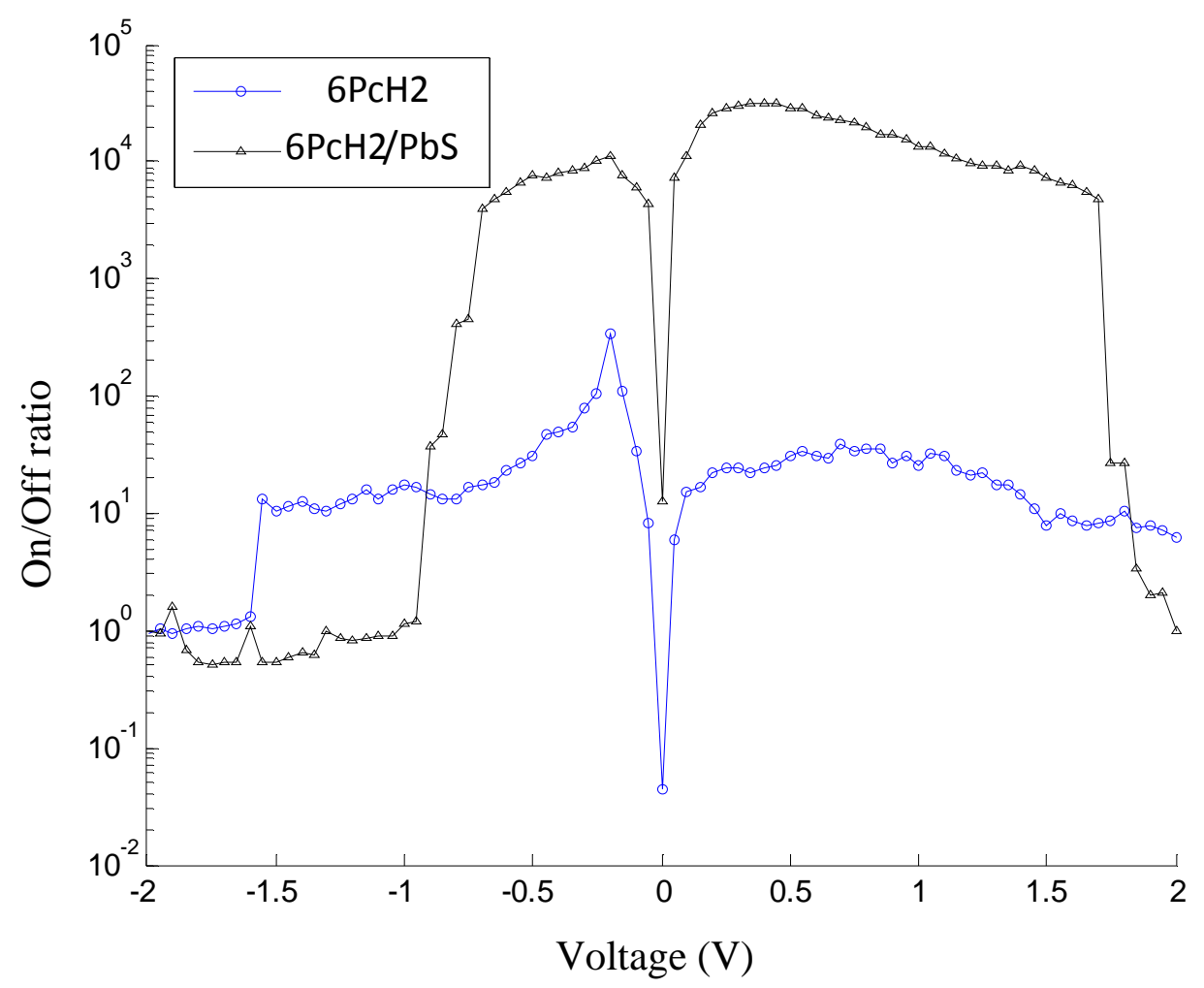

Figure 7 The on/off ratio of two-terminal switches containing $6 \mathrm{PcH} 2 / \mathrm{PbS}$ nanocomposite. (triangles) and pristine $6 \mathrm{PcH} 2$ film (circles). 

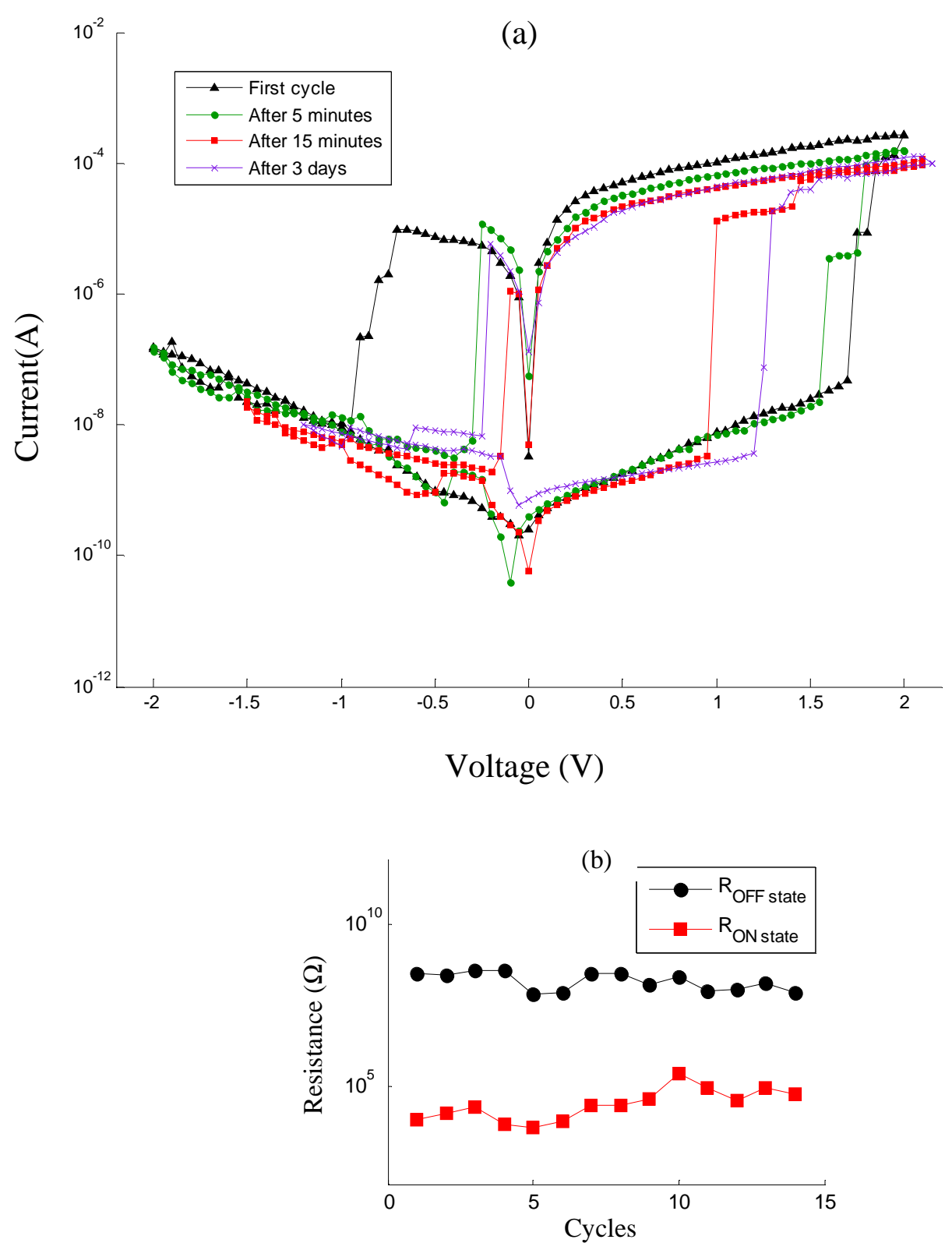

Figure 8 (a) Bistable behaviour of $6 \mathrm{PcH} 2 / \mathrm{PbS}$ nanocomposite for the first cycle (triangle), after 5minutes (circle), 15 minutes (square) and 3 days (cross); (b) Endurance characteristic of $6 \mathrm{PcH} 2 / \mathrm{PbS}$ nanacomposite (solid rectangles and circles for $\mathrm{On}$ and Off states respectively. 